\title{
Correction to: Gluing Compact Matrix Quantum Groups
}

\section{Daniel Gromada ${ }^{1}$}

Accepted: 10 December 2020/ Published online: 8 February 2021

(C) The Author(s) 2021

\section{Correction to: Algebras and Representation Theory https://doi.org/10.1007/s10468-020-10010-0}

The original version of this article unfortunately contains mistakes introduced during the production phase. The equation found in page 21 is incorrect. Below is the correct presentation of the said equation.

$$
\beta:\left(C(H) \otimes_{\max } C^{*}\left(\mathbb{Z}_{k}\right)\right) *_{\mathbb{C}} C^{*}\left(\mathbb{Z}_{l}\right) \rightarrow M_{d}\left(C(H) *_{\mathbb{C}} C^{*}(\mathbb{Z})\right)
$$

Also, the author names of reference 17 and 18 found in the Reference list are incorrect.

17. Malacarne S.: Woronowicz Tannaka-Krein duality and free orthogonal quantum groups. Math. Scand. 122(1):151-160 (2018). https://doi.org/10.7146/math.scand.a-97320

18. Neshveyev S., Tuset L.: Compact Quantum Groups and Their Representation Categories. Société Mathématique de France, Paris (2013) are the corrected entry.

The original article has been corrected.

Publisher's Note Springer Nature remains neutral with regard to jurisdictional claims in published maps and institutional affiliations.

The online version of the original article can be found at https://doi.org/10.1007/s10468-020-10010-0

Daniel Gromada

gromada@math.uni-sb.de

1 Fachbereich Mathematik, Saarland University, Postfach 151150, 66041 Saarbrücken, Germany 